\title{
MASSIVE PROSTHESES FOR MALIGNANT BONE TUMOURS OF THE LIMBS
}

\section{S. R. CANNON}

From the Royal National Orthopaedic Hospital Trust, London, England

The goals of surgeons who treat primary bone tumours of the arms and the legs are the survival of the patient and the salvage of a functional limb. Various methods of limb salvage have been used for more than a century for benign and low-grade malignant tumours. Since the early 1980s, advances in neo-adjuvant chemotherapy have led to an extension of these methods to high-grade sarcomas of bone. Reconstructive operations include the use of autografts, allografts, prosthetic implants, and modified amputations.

The most frequent method is the use of a massive endoprosthesis which may be modular or custom-built. A typical custom-made implant for use in a proximal tibial and knee resection is shown in Figure 1; it is designed for intramedullary cementing, which is the most common method of fixation.

When considering whether surgery for limb-salvage is justified, it is usual to consider long-term oncological results and compare them with the historical results after amputation. Comparisons can be made in four broad areas:

1) the overall survival of patients;

2) the early and late morbidity for each type of reconstruction;

3) the function of the salvaged limb and its maintenance over a prolonged period of follow-up; and

4) the quality of life in patients having limb salvage and those having an amputation.

\section{OVERALL SURVIVAL}

There have been a number of reports from single institutions ${ }^{1,2}$ that surgery for limb salvage had no effect on the long-term survival of patients. These early reports have been confirmed by a number of multi-centre reviews ${ }^{3-5}$. The rate of local recurrence for patients undergoing aboveknee amputation for malignant bone tumours around the knee is about $10 \%$, which is similar to that for patients having limb-salvage surgery ${ }^{4}$. No patient suffered local recurrence after a hip disarticulation, but at the cost of considerable mutilation. Simon ${ }^{6}$ reported a study of the

S. Cannon, Consultant Orthopaedic Surgeon

The London Bone Tumour Service, Royal National Orthopaedic Hospital Trust, 45-61 Bolsover Street, London W1P 8AQ, England.

Printed with the permission of EFORT. This article appears in European Instructional Course Lectures Vol. 3, 1997.
Musculoskeletal Tumor Society in which the development of local recurrence appeared to be an extremely bad prognostic factor for survival; 16 of 17 patients who developed local recurrence after limb salvage or amputation eventually died from their disease. Local recurrence usually requires amputation if there is no other metastatic disease. Even after amputation survival is still unlikely, but it is not clear whether recurrence results from a diminished response of the tumour to neo-adjuvant chemotherapy, and hence a worse prognosis, or to inadequate surgery. In many cases, particularly of late local recurrence not associated with metastatic disease, additional procedures for limb salvage may be considered.

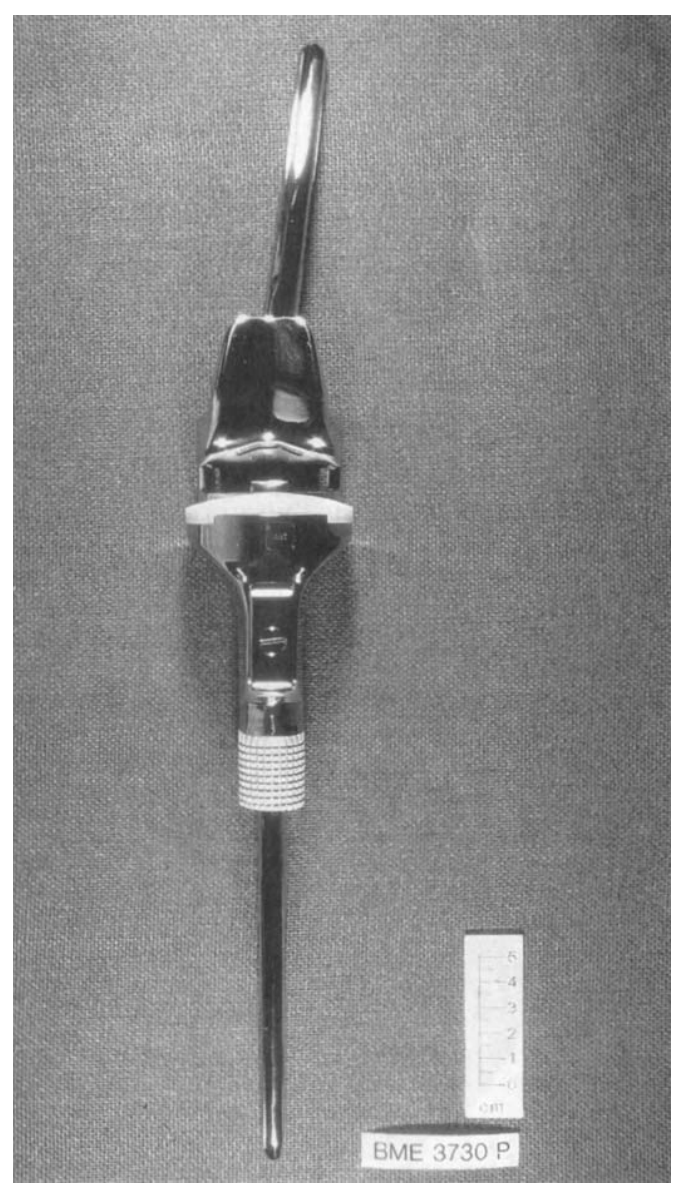

Fig. 1

A typical custom-made proximal tibial implant designed for intramedullary cementing. 


\section{EARLY COMPLICATIONS}

Surgery performed for limb salvage has many complications. There is an occasional acute vascular injury, and venous thrombosis and pulmonary embolism may occur in the early postoperative period. Tumour involvement may require the sacrifice of a nerve during resection, particularly the circumflex and common peroneal nerves, but the most worrying early complication is wound necrosis and subsequent infection.

Loss of cutaneous tissue requires urgent soft-tissue repair, by a local skin flap, or rarely by a myocutaneous free flap, so that the reconstruction can be covered. If infection follows, especially when an endoprosthesis has been used, the outcome may be devastating, and the limb may require amputation in the short or medium term. The onset of infection also delays any planned return to adjuvant chemotherapy. Amputation also has short-term and long-term complications: local pressure sores from an external prosthesis, phantom pain, and overgrowth of the stump in children are all well-recognised.

Endoprostheses are now used for many benign and malignant conditions. There are stronger indications for their use in malignancy when survival is in some doubt and longevity may be limited. Complex reconstruction is rarely justified in this situation. Osteoarticular allografting and bone transportation may be considered in patients with benign or low-grade malignant disease, but in some cases the use of endoprostheses may be indicated. In our practice, this is usually for periarticular destructive lesions, the most common of which is recurrent giant-cell tumour. The frequency of the use of prostheses for different tumours is shown in Figure 2 and Table I; under 20\% of our patients had a benign condition.

Most of the malignant tumours in bones and joints have a predilection for the lower limb, with a few cases in the upper humeral metaphysis. It is therefore not surprising that over $80 \%$ of our endoprostheses were used in the lower limb, with the proximal humerus as the next most common site in the upper limb (Table I). The most commonly

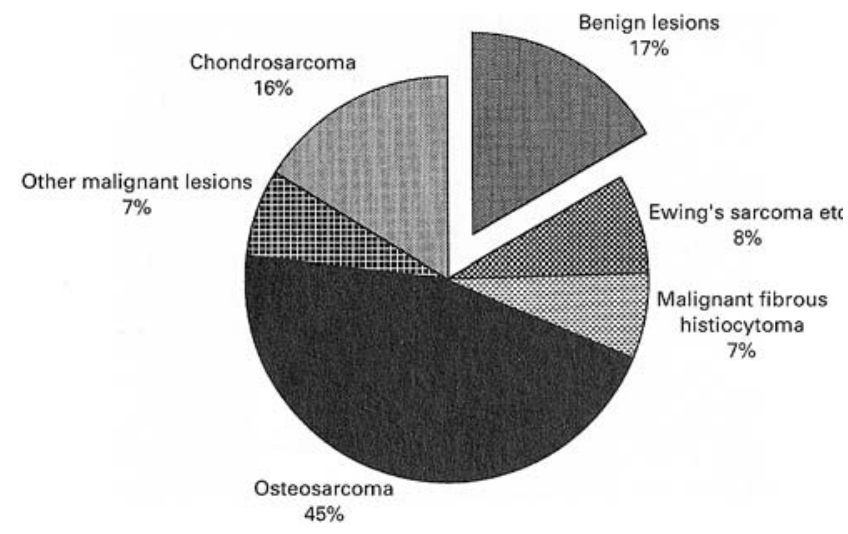

Fig. 2

Diagnostic categories for patients undergoing prosthetic replacement. replaced areas in order of frequency were the distal femur and knee, the proximal femur and hip, the proximal tibia and knee, and the proximal humerus and shoulder. The diaphyses of the femur, tibia and humerus are occasionally replaced, but these represent only a few cases with a similar low proportion of replacement of a whole bone and its adjacent joints.

\section{Illustrative cases}

Each region provides particular problems; these are illustrated by four cases.

Case 1. Proximal humerus. A 12-year-old boy had a fiveweek history of discomfort in the left shoulder before he fell and sustained a shoulder fracture. Investigations showed a pathological fracture through the left proximal humerus with no metastatic disease (Fig. 3). Biopsy confirmed a high-grade osteosarcoma and neo-adjuvant chemotherapy was given. The patient responded, and six weeks after the diagnosis, had resection of the upper left humerus with endoprosthetic replacement (Fig. 4).

The technical problems of upper humeral replacement for such a large tumour are twofold. The first is the proximity of neurovascular structures; the circumflex nerve is particularly vulnerable as it winds round the surgical neck of the humerus. The second problem is possible intracapsular involvement of the glenohumeral joint. Extra-articular resection is rarely adequate; in this patient intra-articular excision was performed. There is debate as to reconstruction of the residual rotator cuff. Early attempts to preserve function by sewing the cuff to a terylene sleeve produced abrasion debris and sinus formation. Some surgeons use such a cuff as an artificial capsule over which the rotator cuff is repaired, but this may lead to considerable stiffness of the glenohumeral joint. Prosthetic replacement of the upper humerus allows excellent restoration of elbow and hand movement, but shoulder function is often very limited. The usual outcome is that rotation is well preserved and controlled, although external rotation may be voluntarily grossly exaggerated. Flexion and abduction are rarely better than $40^{\circ}$, and if the circumflex nerve has been sacrificed there may be none. In manual workers in whom nerve excision is anticipated, a biological or prosthetic arthrodesis of the glenohumeral joint may be a better choice of treatment. Even when the circumflex nerve can be preserved, rotator-cuff function remains poor, and prostheses often sublux into the subacromial space.

Case 2. Proximal femur. A 60-year-old man with a one-

Table I. Relative numbers of sites of endoprosthesess inserted by the London Bone Tumour Service since 1985

\begin{tabular}{lcc}
\hline Site & Number & Percentage \\
\hline Distal femur & 320 & 44 \\
Proximal femur & 142 & 19 \\
Proximal humerus & 85 & 12 \\
Proximal tibia & 140 & 18 \\
Other & 50 & 7 \\
\hline
\end{tabular}

THE JOURNAL OF BONE AND JOINT SURGERY 
month history of pain and tenderness in the upper left thigh had a lucent area in the left proximal femur which was further evaluated by MRI (Fig. 5). Percutaneous needle biopsy under local anaesthesia confirmed a primitive neuroectodermal tumour (PNET), but there were no metastases on further staging. A prolonged course of neoadjuvant chemotherapy caused severe morbidity, but the patient survived and six months after diagnosis had resection of the proximal left femur with endoprosthetic replacement (Fig. 6a).

Intra-articular resection is usually adequate for lesions of the proximal femur. In younger patients a bipolar hip prosthesis is usually preferred, but this patient had total hip replacement and an upper femoral prosthesis. This involved detachment of the iliopsoas and all three gluteal muscles but it was possible to leave tensor fascia lata intact and innervated. Most of the vastus muscles remain innervated, although vastus lateralis may be denervated and vastus intermedius is excised with the tumour.

Two methods of reconstruction of the abductor apparatus are possible. The more usual is soft-tissue weaving of all structures on to the tensor fascia lata. Abduction can be maintained, but the altered lever arm means that many patients have a Trendelenburg limp. The other technique turns down part of the ilium attached to the anterior fibres of gluteus medius, but may lead to fibrosis of the musculature on the outer side of the prosthesis. The most frequent complication is hip dislocation and the patient is advised to use a PoHo-type brace for the first three months after surgery (Fig. 6b).

Case 3. Distal femur. An eight-year-old girl who had a two-week history of pain in her right distal femur awoke one morning with excruciating pain after a tussle with her sibling. She had an undisplaced pathological fracture through an osteosarcoma (Fig. 7). Percutaneous needle biopsy under general anaesthesia showed a chondroblastic osteosarcoma. After six weeks of neo-adjuvant chemotherapy, resection of the tumour was performed. The resected specimen showed that $90 \%$ of the tumour had undergone complete necrosis. After discussion with her parents, the limb was reconstructed using an uncemented extendible prosthesis (Fig. 8). Postoperative recovery was good and the child is now walking without a limp.

Distal femoral prostheses are the most successful in

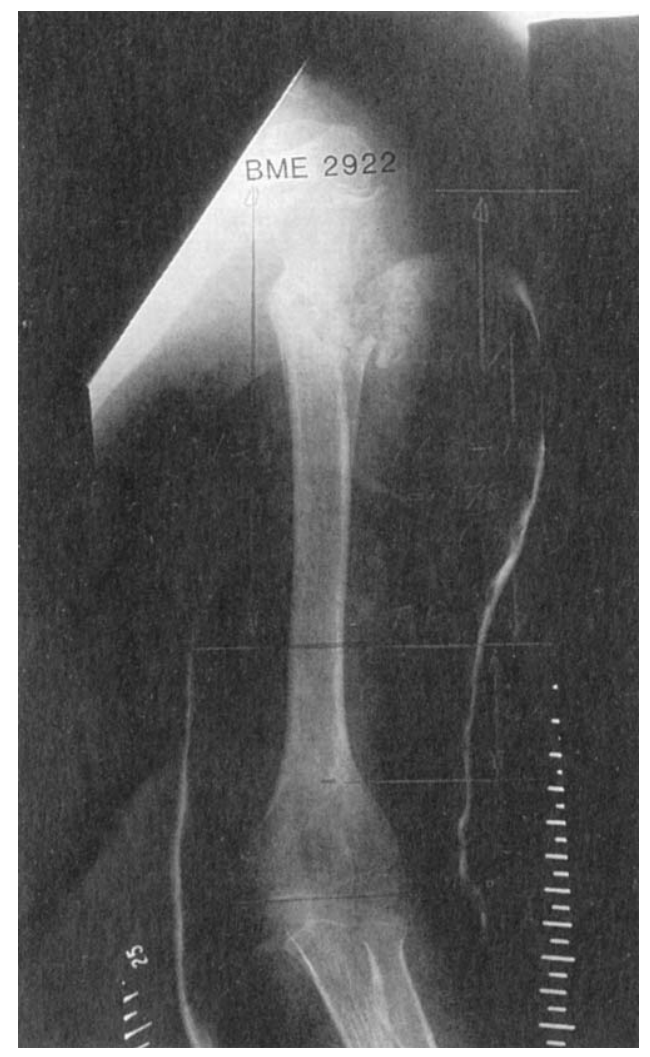

Fig. 3a

Figure 3 - Radiographs of a pathological fracture through an osteosarcoma of the upper humerus (a) and MRI of an upper humeral osteosarcoma (b,c).

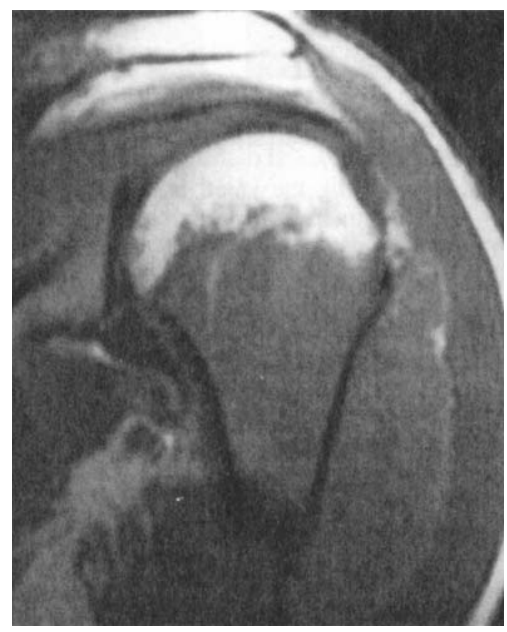

Fig. 3b

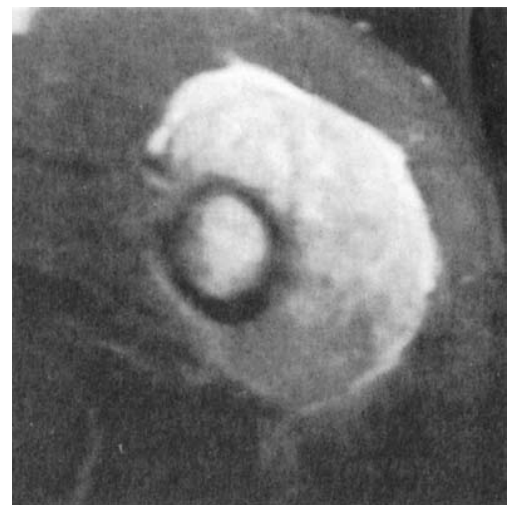

Fig. 3c

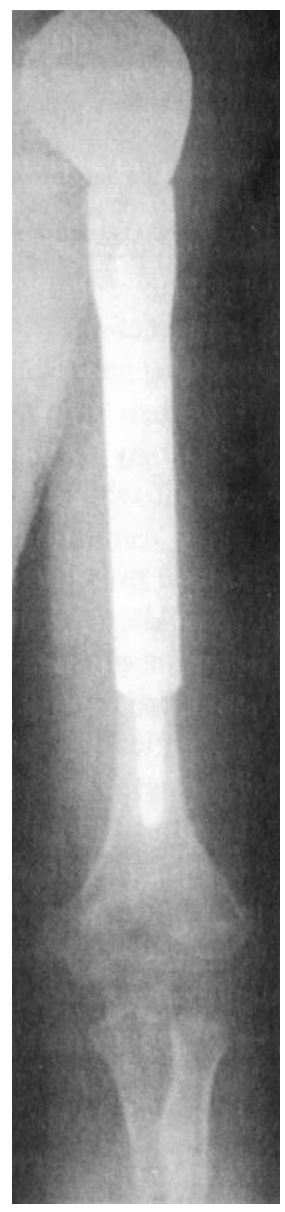

Fig. 4

An upper humeral endoprosthesis. 


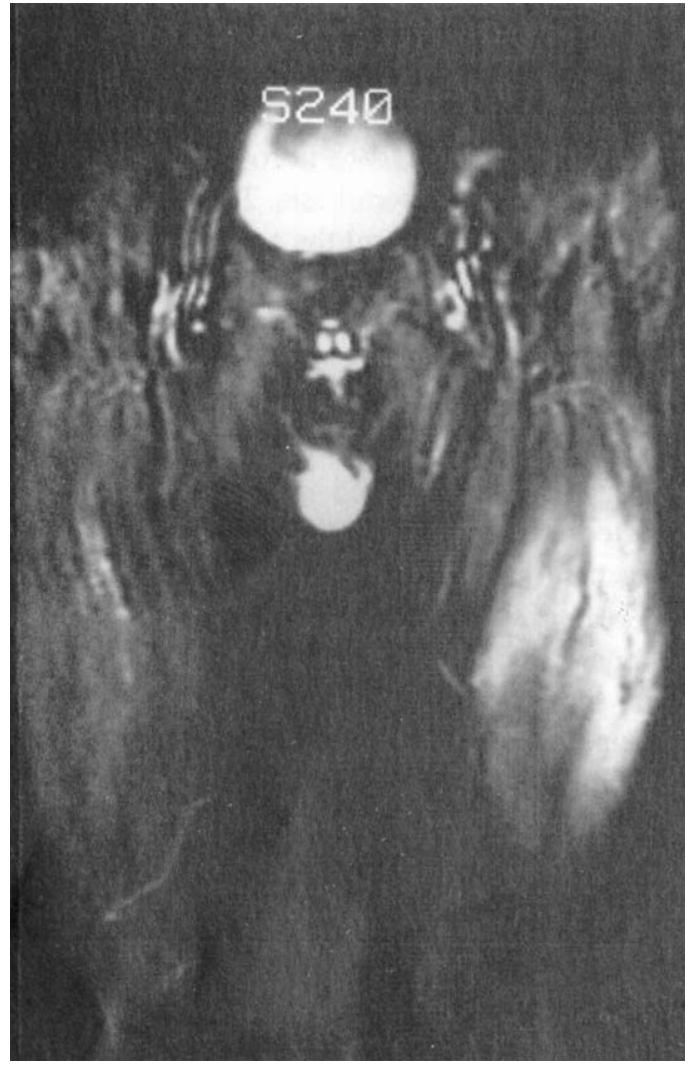

Fig. 5

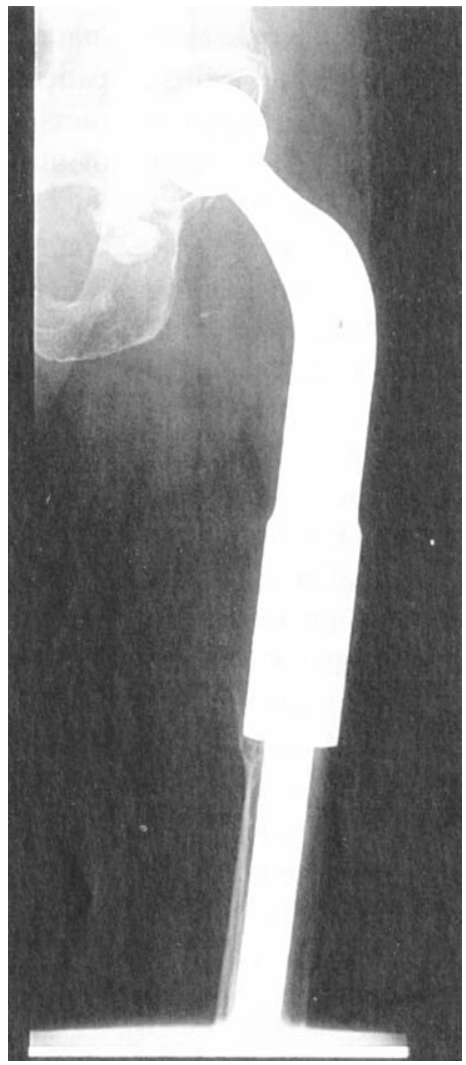

Fig. 6a

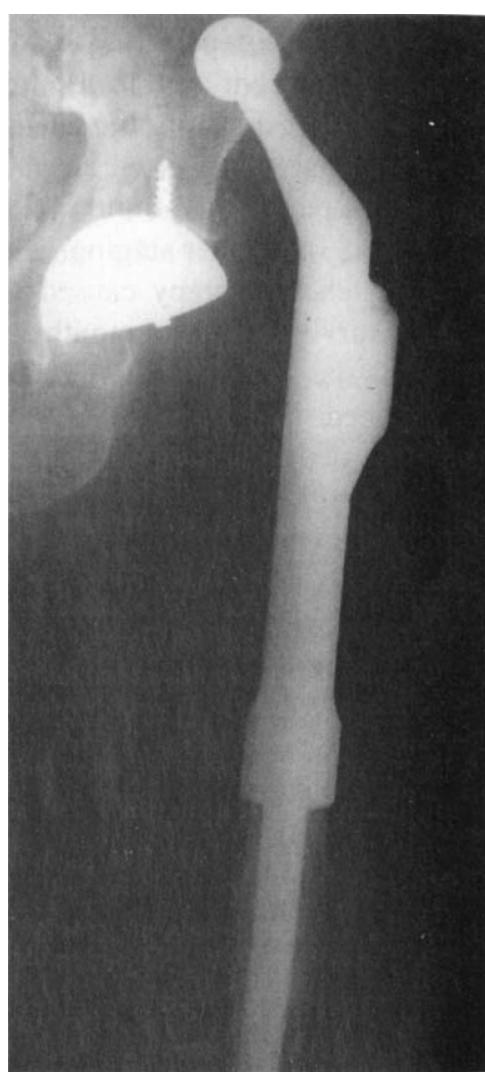

Fig. $6 b$

Figure 5 - MRI of the left proximal femur showing the small bony focus of a PNET tumour with a large soft-tissue component. Figure 6 - An endoprosthetic replacement of the upper femur (a) and a hybrid endoprosthetic replacement of the upper femur with an uncemented acetabular shell and gluteal turn-down reconstruction, showing early postoperative dislocation (b).

terms of early function. Most of the extensor apparatus can be preserved; the gastrocnemei are severely weakened by detachment of their origins, but knee flexion can be fully compensated for by the hamstrings. It may be difficult to tell from the gait which side has been treated.

In this case a minimally invasive method of active elongation of the prosthesis was used. Elongation is by the insertion of an Allen key to turn a low-geared mechanism in the body of the prosthesis. It is, however, likely that the patient will require revision of the prosthesis at a later date, and perhaps surgical growth arrest in the opposite limb to achieve limb-length equality at maturity.

Case 4. Proximal tibia. A 17-year-old boy had a rapidly enlarging swelling of his left proximal tibia, but denied any pain. Plain radiographs showed a lytic area of the upper tibia through an area of abnormal bone. Jamshidi needle biopsy under local anaesthesia confirmed an osteoblastic high-grade osteosarcoma and MRI showed a very large soft-tissue mass extending into the anterior compartment (Fig. 9). Further staging investigations showed no metastatic disease. The patient had neo-adjuvant chemotherapy which was uncomplicated, and six weeks after diagnosis had prosthetic replacement of the right proximal tibia.

Surgical resection of the proximal tibia has many complications, the most important of which is due to the detachment of the extensor mechanism. The next most frequent problem is the common peroneal nerve, which usually has to be mobilised and occasionally sacrificed during the resection of the tumour. The vascular structures are close to the posterior aspect of the knee and the anterior tibial artery is nearly always excised since it is grossly adherent to the tumour as it enters the anterior compartment. The early morbidity is usually due to a combination of ischaemia, compartment syndromes and nerve palsy.

Established nerve palsy can be managed by use of an external ankle-foot orthosis, an ankle arthrodesis or, when appropriate, tendon transfer. In the long term, the major functional disability is caused by weakness of the extensor mechanism. All upper tibial replacements require a medial gastrocnemius flap, which further weakens the flexors of the knee. The extensor mechanism may be reconstructed by simple suture to the transposed medial head of gastrocnemius, but some surgeons prefer transposition of the upper fibula, if it has been retained, by multiple osteotomy or a turn-down of a portion of the distal patella (Fig. 10).

Rehabilitation is slow, with splinting in extension for six to eight weeks. The reconstruction of the extensor mechanism usually stretches, and the patient often develops patella alta and occasionally, some instability. Few patients can achieve a straight-leg raise, but most have a normal walk- 


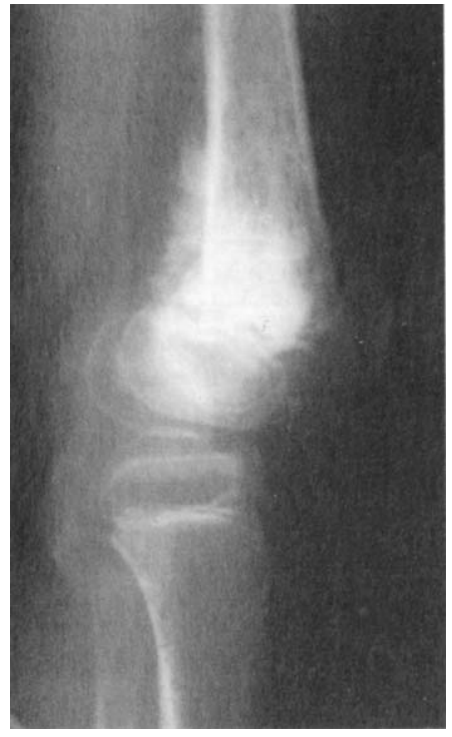

Fig. 7a

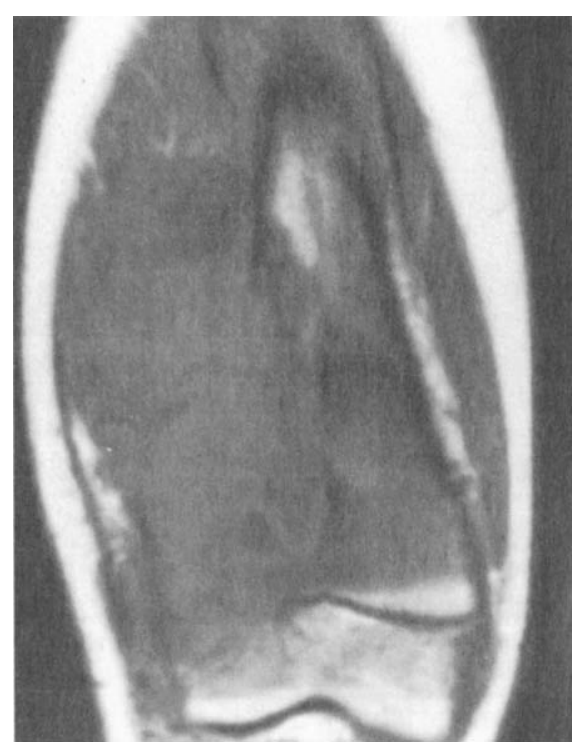

Fig. $7 b$

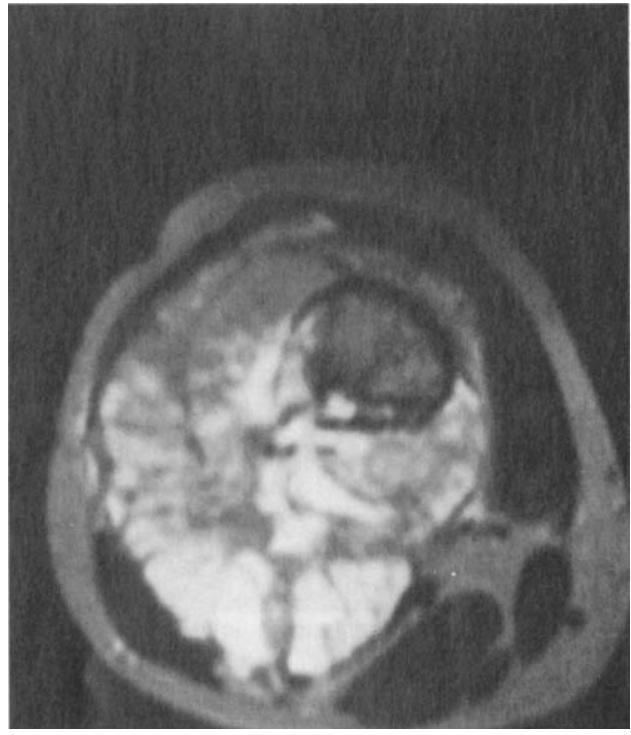

Plain radiograph of a distal femoral osteosarcoma (a), and postchemotherapy MRI views of a distal femoral tumour (b).

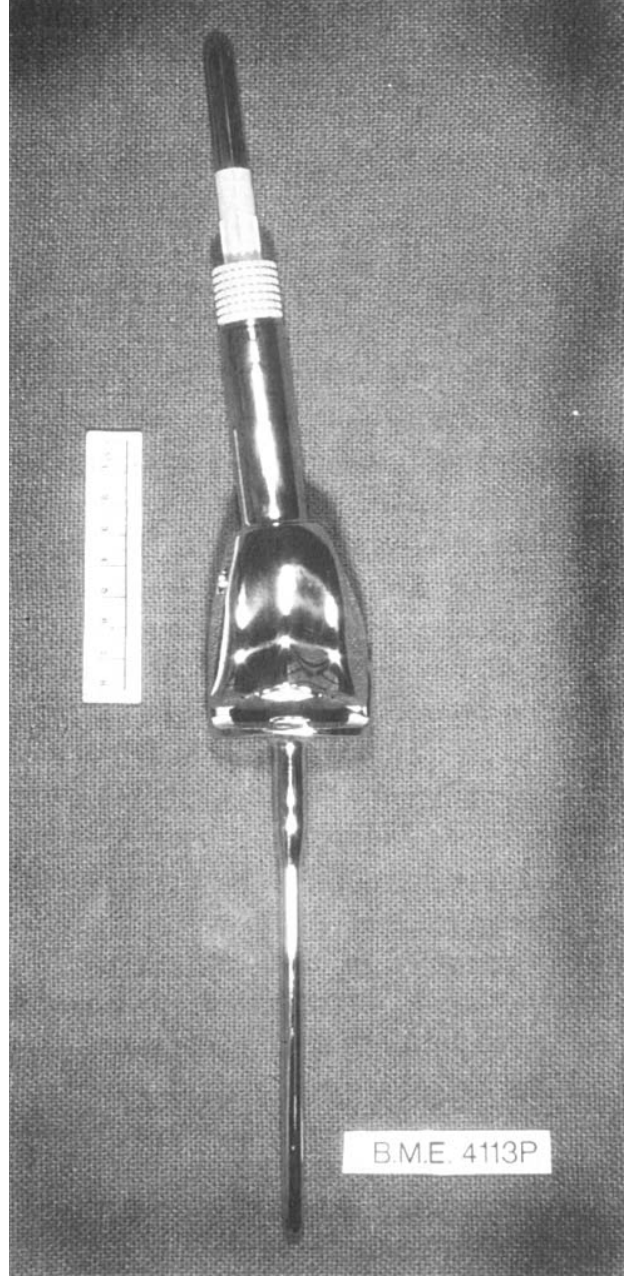

Fig. 8

An uncemented extendible prosthesis. ing pattern. They learn to throw the tibia forwards after hamstring relaxation and then lock the prosthesis in slight hyperextension.

\section{LATE COMPLICATIONS}

The long-term complications of limb salvage depend on the type of reconstruction. Good long-term function after knee arthrodesis or rotationplasty has been reported by Kotz and Salzer, but the first method abolishes movement at the knee and the second mutilates the limb. The long-term incidence of aseptic loosening after implantation of a cemented custom endoprosthesis into the lower limb has been reported by Unwin et $\mathrm{al}^{8}$ after the clinical and radiological follow-up of 493 distal femoral, 263 proximal femoral, and 245 proximal tibial implantations. Upper humeral prostheses, which are non-weight-bearing, all produce satisfactory function and aseptic loosening is rare.We excluded these from our study of revision.

\section{Review of 1001 cases}

Revision for aseptic loosening was required in 71 of the total of 1001 patients, giving an incidence of $7 \%$ in the only long-term outcome report. Earlier studies on cemented endoprostheses by Scales and Wright ${ }^{9}$ showed an aseptic loosening rate of $1.9 \%$ and Bradish et al, ${ }^{10}$ reviewing only distal femoral replacements, found no incidence of aseptic loosening. Roberts et al $^{11}$ studied 135 distal femoral replacements after bone-tumour resection and reported aseptic loosening in 6\%; infection was of greater concern with an incidence of $6.8 \%$.

It is recognised that the incidence of infection is greater after proximal tibial replacement than in other regions. Modification of the surgical technique, using a medial gastrocnemius flap in all cases, has reduced the incidence 


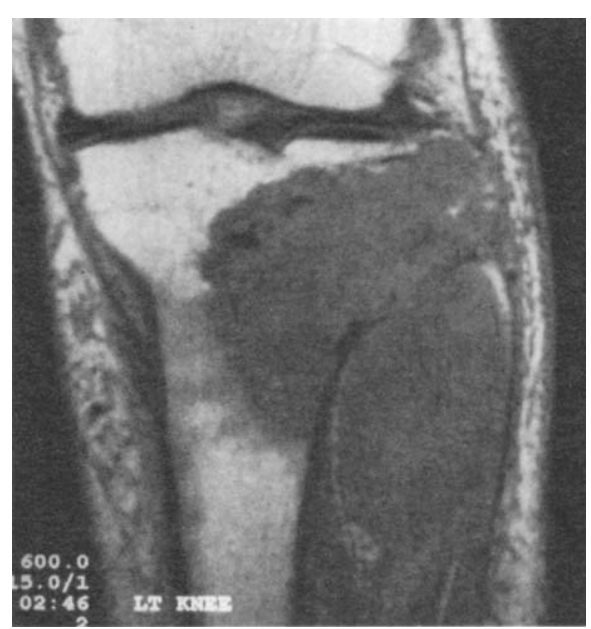

Fig. 9a

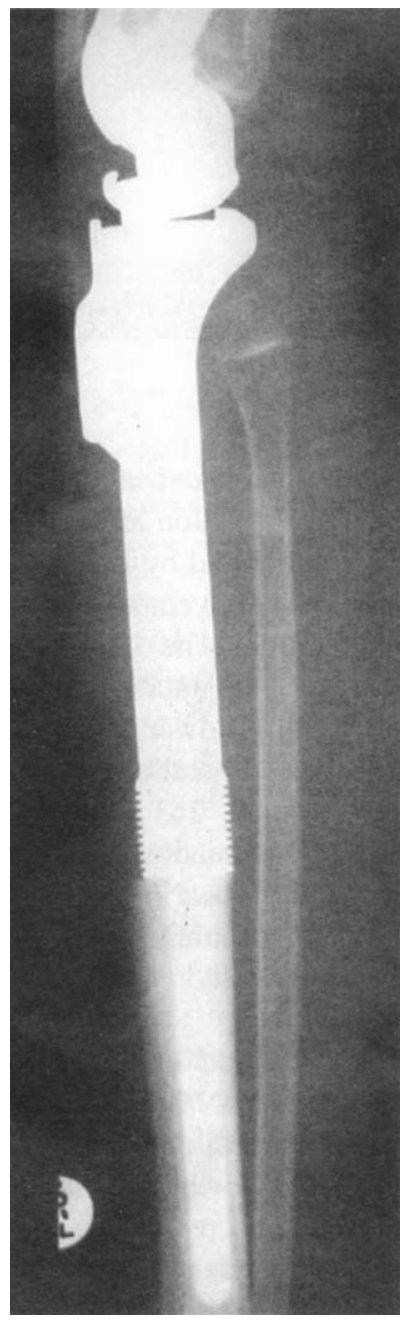

Fig. 10a

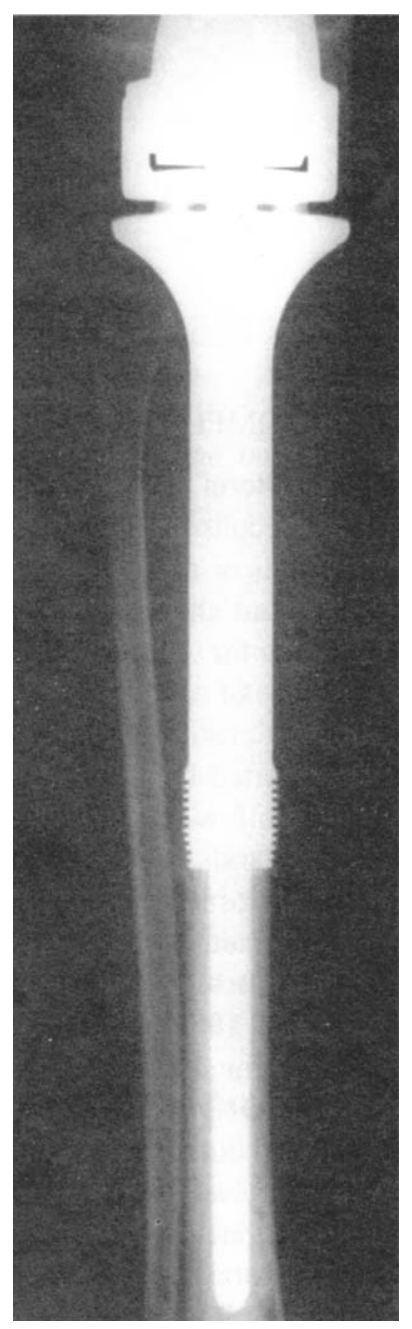

Fig. 10b
An upper tibial prosthesis showing the area designed for quadriceps reinsertion by patellar turn-down.

of sepsis as reported by Grimer et $\mathrm{al}^{12}$; aseptic loosening is now the principal mode of failure for cemented massive

Fig. $9 b$

MRI of an osteosarcoma of the upper left tibia.

prostheses. Capanna et $\mathrm{al}^{13}$ noted that aseptic loosening is extremely rare in uncemented implants, but had some cases of implant fracture in addition to some infections.

In our series the predominant indications for limb-salvage were osteosarcoma (44.7\%), osteoclastoma (12.8\%) and chondrosarcoma (12.6\%) (Fig. 11). The average age of the patients was 29.9 years, but with considerable variation in the three groups. The youngest patient had a proximal femoral replacement at three years of age and the oldest had proximal femoral and distal femoral replacements at 84 years of age. The youngest group were those having proximal tibial replacement $(23.3 \pm 2.2$ years $)$ and the oldest had proximal femoral prostheses $(41.5 \pm 2.1$ years $)$.

All our endoprostheses are individually designed and custom-made at the Department of Biomedical Engineering, Stanmore (University of London). The body and intramedullary stems are of titanium alloy and curved to follow the natural anatomy of the shaft of the femur. The femoral head is of cobalt-chrome molybdenum. The intramedullary stems in the tibia were straight with a fixed hinge of cobalt chrome at the knee.

We calculated the percentage of bone excised from measurement on anteroposterior radiographs. In each of the proximal femoral, distal femoral and proximal tibial groups, the mean amount resected was $50 \%$. The proximal femoral replacements had the most bone removed and the proximal tibial replacements the least, but the differences were small.

The overall outcome was analysed by age at the time of surgery: under 20 years, 20 to 60 years and over 60 years. The percentage of bone resected was recorded as: less than $40 \%, 40 \%$ to $60 \%$, or over $60 \%$. The end-point for the study was when the first prosthesis was removed irrespective of the subsequent management.

Of all cases, $84 \%$ had a follow-up of over three years, with an average follow-up of nearly four years. The total review was equivalent to 3216 implant years. Amputation had been required in $9 \%$, most for recurrence, and $17 \%$ had died from metastatic disease. Revision procedures were 


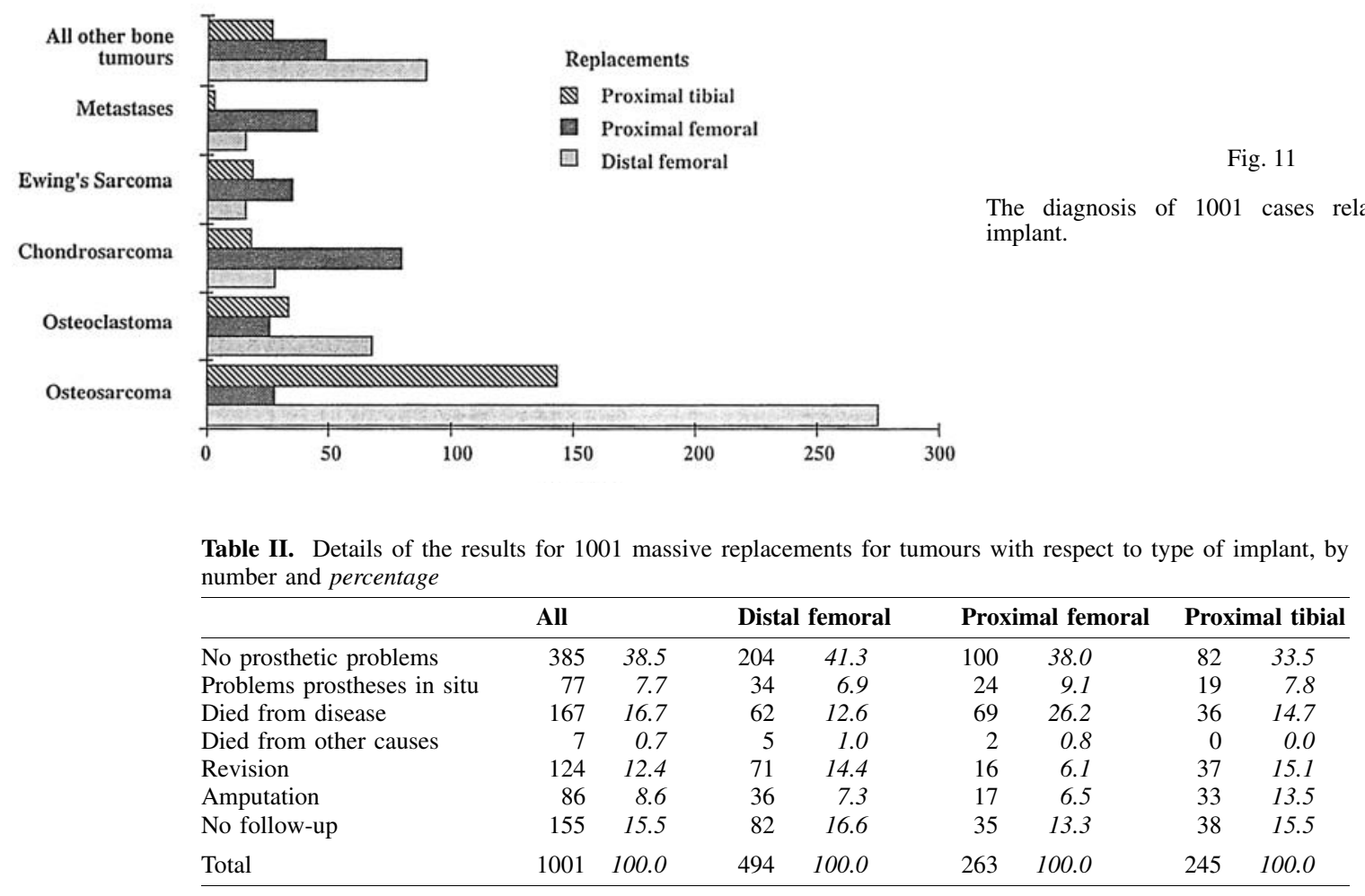

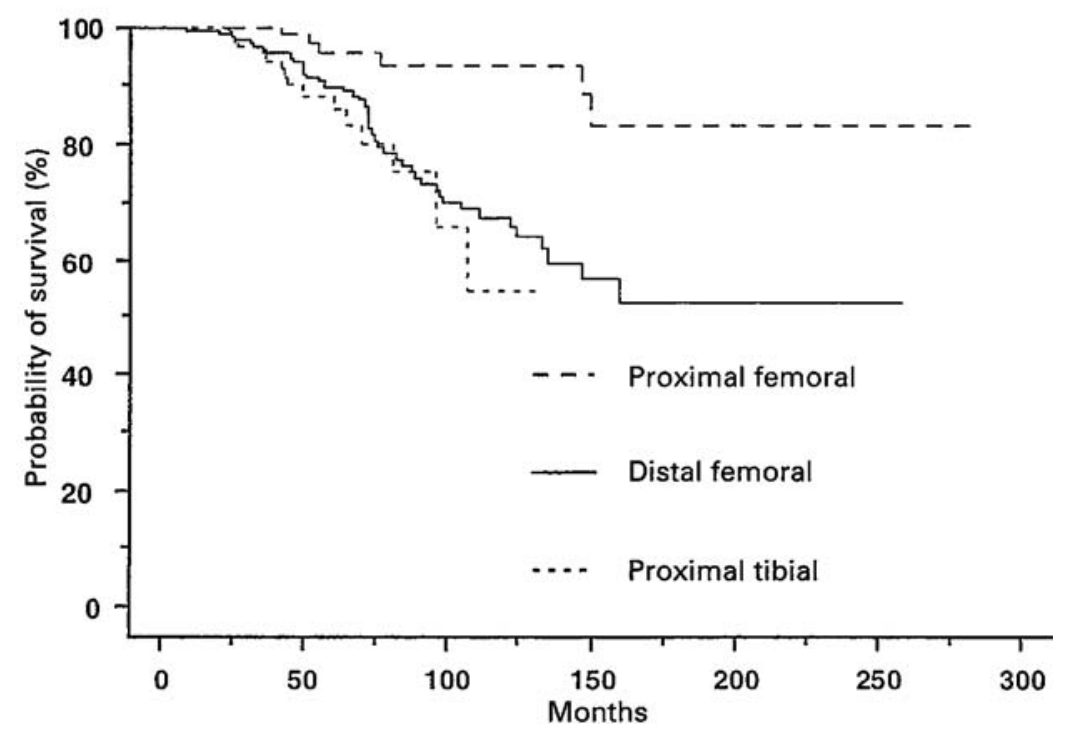

Fig. 12

The probability of surviving aseptic loosening related to the type of implant. needed for 124 patients, 71 of them for aseptic loosening. This was most common after distal femoral replacement; reoperation on the proximal tibia was most often for infection. Details of the reoperations are shown in Table II.

In the 71 cases of revision for aseptic loosening of an intramedullary stem most were revised with a second custom-made endoprosthesis. Failure as a result of aseptic loosening was seen in $9.9 \%$ of distal femoral replacements, but only $2.3 \%$ of proximal femoral implants required revision for this cause.

The rates of aseptic loosening also varied in relation to age, probably reflecting the different aetiology in different regions: osteosarcoma is seen in a younger age group and around the knee; chondrosarcoma occurs in older patients and in the proximal femur. For similar reasons the distal femoral group was the most likely to be given chemotherapy.

There was also considerable variation in survivorship in relation to the percentage of bone removed. In the distal femoral and proximal tibial groups, removal of a small percentage of the bone gave a low failure rate; when more than $60 \%$ was removed the failure rate was much higher. In the proximal femoral group, this relationship was reversed. 
Our series as a whole showed an extremely low probability of aseptic loosening in the first three years. Figure 12 shows that proximal tibial replacements had the worst prognosis. The probability of avoiding aseptic loosening for ten years was $67.4 \%$ in the distal femoral group, $54.8 \%$ in the proximal tibial group and $93.8 \%$ in the proximal femoral group. The effect of age at the time of operation had the greatest influence on patients under 20 years after distal femoral replacement (Fig. 13). When age and the amount of bone resected were both taken into account, patients under 20 years with more than $60 \%$ of the distal femur or the proximal tibia resected had the poorest outcome (Table III).

\section{DISCUSSION}

The most interesting questions arising from our study are why distal femoral and proximal tibial replacements give worse results than proximal femoral replacements and why age at operation and the percentage of bone removed influence survival? In the two femoral groups, an important mechanical difference is the amount of off set of the tip of the prosthesis from the weight-bearing axis from the centre of the femoral head to the centre of the knee (Fig. 14). Telemetric studies by Taylor et $\mathrm{al}^{14}$ have shown that, in a cemented prosthesis, most of the force is transmitted rapidly to the tip of the prosthesis. A rise in off set will increase the bending moment around this and this may explain the greater loosening of distal femoral replacements. A second reason may be the torsional moment around a fixed-hinge knee replacement in comparison with that on an unconstrained hip. Since 1991, we have used a rotating-hinge design for distal femoral and proximal tibial replacements; the early results show a dramatic improvement in the rate of aseptic loosening.

We now have new telemetric data from a 42-year-old patient with a distal femoral replacement using a rotatinghinge at the knee. This showed that axial forces were between 2 and $2.8 \times$ body-weight during walking, ascend- ing and descending stairs, and rising from a chair. The force at the tip of the prosthesis, measured as a tip-to-shaft ratio, was $11 \%$ at six months and $22 \%$ at nine months, since when it has remained stable. In a proximal femoral replacement without a hydroxyapatite (HA)-coated collar, the tipto-shaft ratio of forces increased to $57 \%$ at one year.

Our earlier reviews showed that there was a tendency to the formation of bony collars, particularly on the posterior and medial sides of endoprostheses. We therefore studied the effect of porous collars and HA-coated collars in improving long-term fixation of distal femoral endoprostheses. We used five porous collars and $32 \mathrm{HA}$ collars and compared the findings with 31 uncoated prostheses. Pedicle formation was seen in all three groups, but a there was pedicle gap in $95 \%$ of the control group and in all of those with porous collars. Bone ingrowth was achieved only with HA-coated collars: the incidence of radiolucent lines was greatly reduced in those prostheses fitted with coated collars as reported by Vieten, Blunn and Walker. ${ }^{15}$ We regard it as established practice to insert either a totally uncemented prosthesis with HA-coating of the stem and collar or a cemented prosthesis, fixation of which is supplemented by an HA collar.

The use of endoprostheses in growing children is still contentious. Many surgeons argue that primary amputation gives less morbidity than the multiple surgery required for patients who have an oncologically successful result. It can be argued that patients who have limb salvage and avoid amputation but subsequently die from their disease will have had a better period of life than those who have had surgical mutilation. Given the present level of oncological success for both osteosarcoma and Ewing's sarcoma, the problems which face orthopaedic surgeons who treat growing children with these tumour are increasing. We use a steadily increasing number of extendible prostheses (Fig. 15), but recognise that these are a temporary solution to the problem; nearly all of them require major revision before or at skeletal maturity as reported by Schindler et al. ${ }^{16}$

\section{Function and quality of life}

Table III. The effects of age and the amount of bone resected and implant type on failure by aseptic loosening

\begin{tabular}{|c|c|c|c|}
\hline & $<20$ years old & 20 to 60 years old & $>60$ years old \\
\hline $\begin{array}{l}\text { Proximal femoral } \\
\quad<40 \% \text { resection } \\
40 \% \text { to } 60 \% \text { resection } \\
>60 \% \text { resection }\end{array}$ & $\begin{array}{l}0(10) \\
* *(17) \\
0(18)\end{array}$ & $\begin{array}{l}* *(53) \\
*(88) \\
0(27)\end{array}$ & $\begin{array}{l}0(12) \\
0(34) \\
--(2)\end{array}$ \\
\hline $\begin{array}{l}\text { Distal femoral } \\
\quad<40 \% \text { resection } \\
40 \% \text { to } 60 \% \text { resection } \\
>60 \% \text { resection }\end{array}$ & $\begin{array}{l}*(26) \\
* * *(137) \\
* * * *(66)\end{array}$ & $\begin{array}{l}* *(77) \\
* *(119) \\
* *(21)\end{array}$ & $\begin{array}{l}0(13) \\
0(21) \\
--(6)\end{array}$ \\
\hline $\begin{array}{l}\text { Proximal tibial } \\
\quad<40 \% \text { resection } \\
40 \% \text { to } 60 \% \text { resection } \\
>60 \% \text { resection }\end{array}$ & $\begin{array}{l}0(18) \\
*(91) \\
* * *(33)\end{array}$ & $\begin{array}{l}0(30) \\
* *(55) \\
--(6)\end{array}$ & $\begin{array}{l}--(2) \\
--(7) \\
--(0)\end{array}$ \\
\hline
\end{tabular}



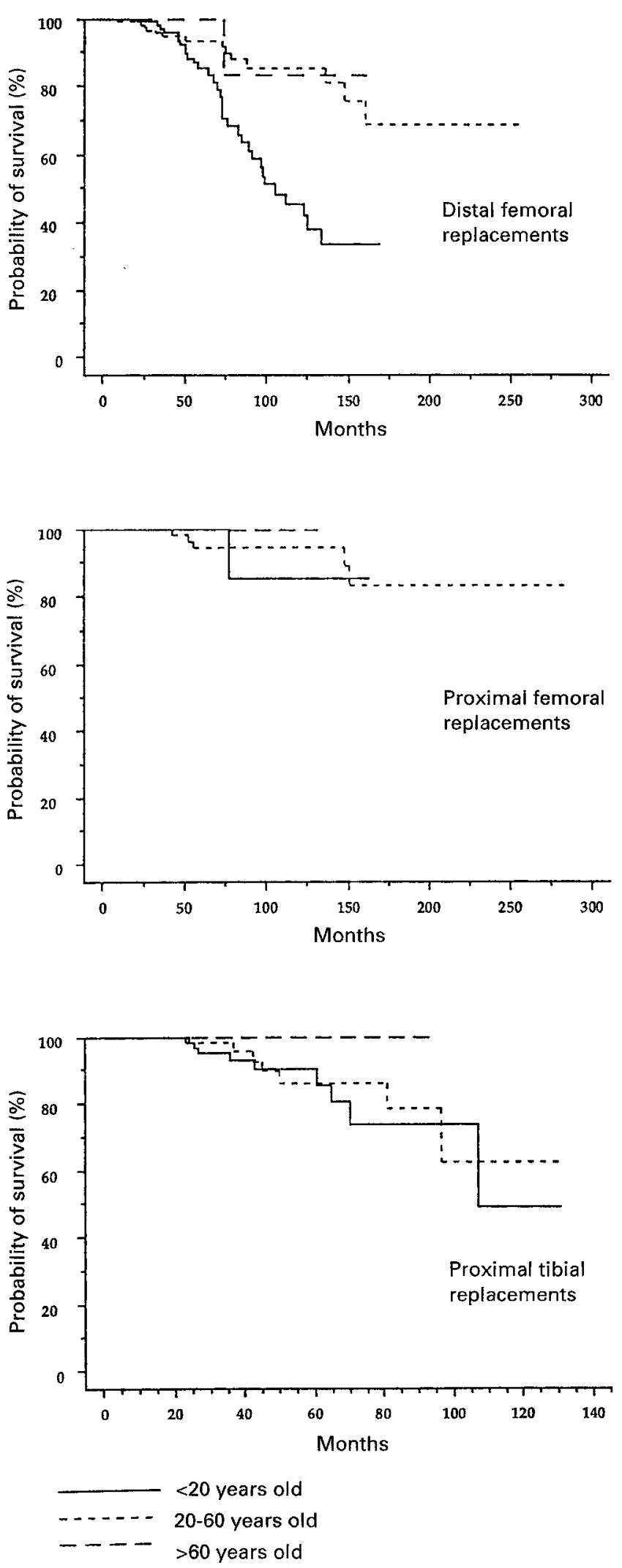

Fig. 13

The probability of surviving aseptic loosening related to the age of the patient.

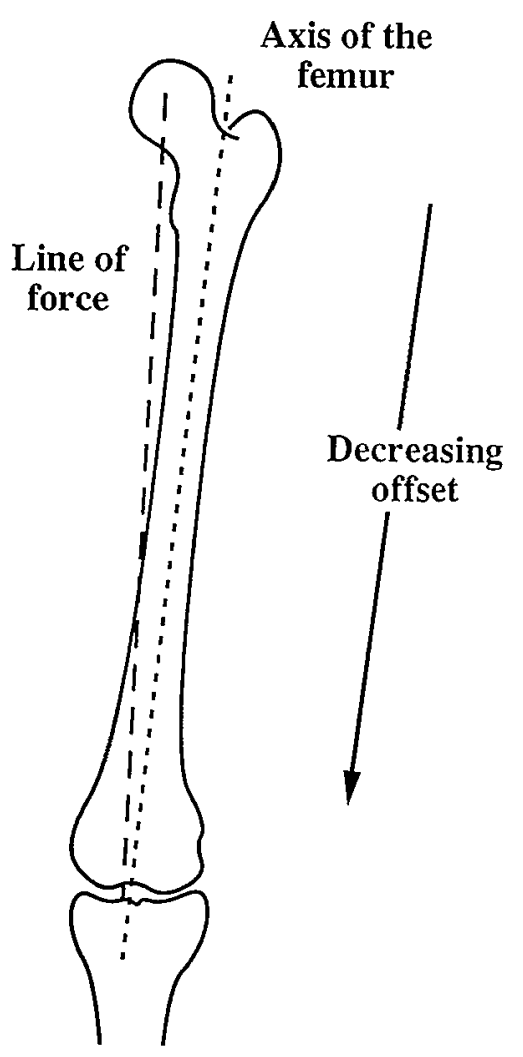

Fig. 14

The influence of offset distance between the line of force and the axis of the femur.

Simon $^{6}$ has argued that an extra-articular resection of the proximal humerus, leaving a flail arm, gives better functional results than a forequarter amputation, and that an arthrodesis of the shoulder using an allograft gives better function than a flail extra-articular resection. In the lower limb, local pelvic resection and reconstruction of the proximal part of the femur will give better results than a hindquarter amputation, although such surgery is often marginal and has many complications including local recurrence.

Most comparisons of methods of reconstruction have discussed the management of the distal femur, proximal tibia and knee. Otis, Lane and Kroll ${ }^{17}$ have assessed the energy levels required to walk after various methods of reconstruction: they concluded that endoprosthetic replacement of the knee and either the distal femur or proximal tibia had lower energy expenditure costs for walking than an above-knee amputation. They also considered that patients with belowknee amputations had lower energy expenditures than those who had prosthetic knee replacements. Energy expenditure during gait, and function after an operation for osteosarcoma are best after mobile replacement of the knee and progressively worse after rotationplasty, arthrodesis of the knee, and above-knee amputation.

The early work of Sugarbaker et al in $1982^{18}$ questioned whether limb salvage and radiotherapy were preferable to 


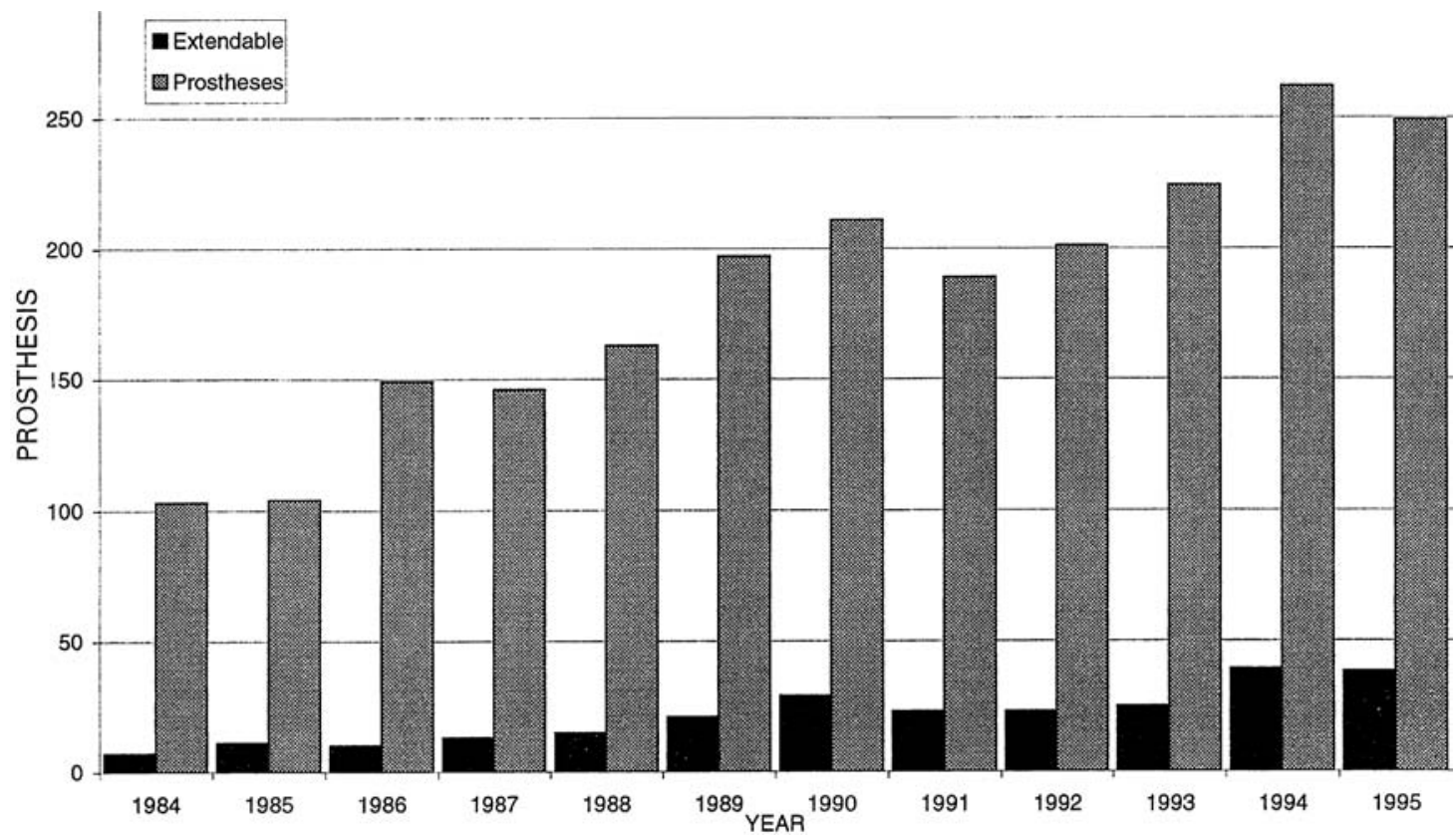

Fig. 15

The numbers of prostheses inserted (standard and extendible) over the last ten years.

amputation. One of the problems is that patients who have salvage procedures often require multiple operations, particularly when an immature skeleton requires extendible methods of reconstruction. Local recurrence or infection will reduce the patient's quality of life, but this is difficult to quantify.

At best, it seems that a patient who does not have a major premorbid psychological disorder will generally make a very good adjustment to whichever operation is chosen; the type of procedure may make little difference. ${ }^{19,20}$

\section{REFERENCES}

1. Sim FH, Ivins JC, Taylor WF, Chao EYS. Limb-sparing surgery for osteosarcoma: Mayo Clinic experience. Cancer Treat Sympos 1985;3:139-54.

2. Lane JM, Glasser DB, Duane K, et al. Osteogenic sarcoma: two hundred and thirty-three consecutive patients treated with neoadjuvant chemotherapy. Orthop Trans 1987;11:495.

3. Winkler K, Beron G, Kotz R, et al. Neoadjuvant chemotherpay for osteogenic sarcoma: results of a co-operative German/Austrian study. J Clin Oncol 1984;2:617-24.

4. Simon MA, Aschiliman MA, Thomas N, Mankin HJ. Limb-salvage treatment versus amputation for osteosarcoma of the distal end of the femur. J Bone Joint Surg [Am] 1986;68-A:1331-7.

5. Bramwell VH, Burgers M, Sneath R, et al. A comparison of two short intensive adjuvant chemotherapy regimens in operable osteosarcoma of limbs in children and young adults: the first study of the European Osteosarcoma Intergroup. J Clin Orthop 1992;10:1579-91.

6. Simon MA. Current concepts review: limb salvage for osteosarcoma. J Bone Joint Surg [Am] 1988;70-A:307-10.

7. Kotz R, Salzer M. Rotation-plasty for childhood osteosarcoma of the distal part of the femur. J Bone Joint Surg [Am] 1982;64-A:959-69.

8. Unwin PS, Cannon SR, Grimer RJ, et al. Aseptic loosening in cemented custom-made replacements for bone tumours of the lower limb. J Bone Joint Surg [Br] 1996;78-B:5-13.
9. Scales JT, Wright KWJ. Major bone and joint replacement using custom implants. In: Chao EYS, Ivins JC, eds. Tumor prostheses for bone and joint reconstruction: the design and application. New York: Thieme-Stratton Inc, Georg Thieme Verlag, 1983:149-68.

10. Bradish CF, Kemp HBS, Scales JT, Wilson JN. Distal femoral replacement by custom-made prostheses. J Bone Joint Surg [Br] 1987; 69-B:276-84

11. Roberts P, Chan D, Grimer RJ, Sneath RS, Scales JT. Prosthetic replacement of the distal femur for primary bone tumors. J Bone Joint Surg [Br] 1991;73-B:762-9.

12. Grimer RJ, Ratcliffe P, Carter SR, Sneath RS. Reduction of infection of proximal tibial endoprostheses. In: Brown KLB, ed. Complications of limb salvage: prevention, management and outcome. Montreal, ISOLS, 1991:331.

13. Capanna R, Ruggeri P, Decristofaro R, et al. Complications, their treatment and outcome in 257 cementless megaprostheses. In: Brown $\mathrm{KLB}$, ed. Complications of limb salvage: prevention, management and outcome. Montreal, ISOLS, 1991:147-50.

14. Taylor S, Perry J, Adler J, Unwin PS, Walker PS. The telemetry of forces in vivo developed in massive orthopaedic implants: the first 18 months, results from walking. In: Tan SK, ed. Limb salvage: current trends. Singapore, ISOLS, 1993:560.

15. Vieten D, Blunn GW, Walker PS et al. Radiographic and histological evaluation of fixation of massive distal femoral endoprostheses by porous coated and hydroxy-apatite coated in-growth collars. Presented at the British Orthop Oncology Society. Autumn 1996.

16. Schindler OS, Cannon SR, Briggs TUR et al. Clinical experience with extendible prosthetic distal femoral replacements for primary malignant bone tumours in the growing individual. Presented $\mathrm{Br}$ Orthop Oncology Soc. Autumn 1996.

17. Otis JC, Lane JM, Kroll MA. Energy cost during gait in osteosarcoma patients after resection and knee replacement and after abovethe-knee amputation. J Bone Joint Surg [Am] 1985;67-A:606-11.

18. Sugarbaker PH, Barofsky I, Rosenberg SA, Gianola FJ. Quality of life assessment of patients in extremity sarcoma clinical trials. Surgery 1982;91:17-23.

19. Boyle M, Tebbi CK, Mindell RE, Mettlin CJ. Adolescent adjustment to amputation. Med Pediatr Oncol 1982;10:301-12.

20. Weddington WW Jr, Segraves KB, Simon MA. Psychological outcome of extremity sarcoma survivors undergoing amputation or limb salvage. J Clin Oncol 1985;3:1393-9. 